\title{
A Comparison of Adaptive Sentence-in-Noise Tests
}

\author{
Jae Hee Lee, Dong Woon Yi \\ Department of Audiology, Hallym University of Graduate Studies, Seoul, Korea
}

\author{
변동형 소음 하 문장인지 평가 도구의 비교 \\ 이 재 희·이 동 운 \\ 한림국제대학원대학교 청각학과
}

\begin{abstract}
Purpose: Daily communication often requires the ability to understand speech in background noise. Given that speech-in-noise understanding is not predictable from speech recognition in quiet and that sentences are more representative of daily communication, different forms (fixed vs. adaptive) of sentence-in-noise tests have been developed and standardized. Relative to the fixed test, the adaptive sentence-in-noise tests are known to be more useful to adjust the difficulty level of the test. Given this advantage, the present review focuses on the features of the adaptive sentence-in-noise tests. Methods: Various types of adaptive sentence-in-noise tests have been developed internationally and standardized for multi-language. This paper compares and summarizes three adaptive sentence-in-noise tests (Hearing in Noise Test, Quick Speech-In-Noise test, and Matrix test) in terms of the three aspects: 1) sources of target sentences and background noise, 2) test procedure and scoring formula, and 3) norms and interpretation of test results. Results: For all the types of adaptive tests, the intensity level of the target sentence or the noise is adjusted based on the listener's response. The sources of target sentences and background noise differ among tests. Various multi-lingual versions of the Hearing in Noise Test and the Matrix test have been developed and reported. All the three tests measure sentence recognition threshold where correct sentence recognition of $50 \%$ occurs. However, the test procedure (i.e., presentation of stimuli/noise, the step size), the scoring formula, and the method to establish norms and interpret the test results depend on the purpose of each test. Conclusion: Compared to other languages, a lack of Korean adaptive sentence-innoise test tools poses challenges for clinical practice and research in Korea. Thus, development of the adaptive Korean sentence-in-noise test which is reliable and efficient is required to quantify speech-recognition abilities of listeners with hearing loss. After developing the adaptive test, the reliability and validity tests need to be continued to estimate the amount of everyday communication difficulty sensitively and to provide better insights into the appropriate amplification and communication strategies.
\end{abstract}

Key Words: Adaptive sentence-in-noise test, Speech perception in noise, Speech audiometry.

Received: October 7, 2016 / Revised: December 5, 2016 / Accepted: December 13, 2016

Correspondence: Jae Hee Lee, Department of Audiology, Hallym University of Graduate Studies, 405 Yeoksam-ro, Gangnam-gu, Seoul 06198, Korea Tel: +82-2-2051-2942 / Fax: +82-2-3453-6618 / E-mail: leejaehee@hallym.ac.kr

\section{INTRODUCTION}

청각장애인의 난청 종류, 정도, 형태 등을 평가하기 위해 순 음을 사용하여 주파수별 청력역치를 측정한다. 그러나 순음청 력역치만으로 실제 의사소통 시 사용하는 다양한 청각적 능력 을 평가하기는 제한적이다(Killion \& Niquette, 2000). 이를 보 완하기 위해 청능사(audiologist)는 어음청각검사를 시행하여 난청인의 어음 인지 능력과 의사소통 장애의 정도를 평가할 뿐 아니라 보장구 착용 혜택을 파악하고 청각재활을 계획하는 데
활용한다(Killion et al., 2004).

미국의 경우 1910년에 Campbell이 전화 채널을 평가하기 위 해 무의미음절 목록을 제작한 것을 시작(Campbell, 1910)으로 다양한 분야에서 인간의 의사소통 능력을 평가하고자 만든 도 구들이 어음청각검사용 도구로 확장되었다. Hudgins et al. (1947)이 녹음한 이음절어 목록은 어음에 대한 민감성을 평가 하는 데 사용되었고, Egan(1948)이 개발한 Harvard Psychoacoustic Laboratory (PAL) 목록은 추후 단음절, 이음절, 문 장, 무의미음절 목록 등으로 확장되었다. Harvard PAL 목록의 
단점을 수정 보완하여 1952년에 Central Institute for the Deaf (CID) W-1, W-2, W-22 목록 등으로 발표되었고(Hirsh et al., 1952), CID W-22의 제한점을 고려하여 Northwestern University Auditory Test No. 6 가 개발되었다. 그 외 학령기 아동을 위 한 Bench-Kowal-Bamford (BKB) 문장 검사 도구(Bench et al., 1979), 일상생활 문장을 이용하는 CID 문장 검사(Silverman \& Hirsh, 1955) 등이 발표되었다.

국내의 경우 1960 년대 당시 빈번하게 사용되었던 단어들로 구성한 단음절어표 $(\mathrm{Hahm}, 1962)$ 가 발표되어 임상에서 사용되 어 왔으나 검사를 위한 표준화 작업까지 마무리되지 않았다. 2005년 국내 조사 연구에 의하면 80개의 전국 수련병원에서 표 준화가 이루어지지 않은 13 종의 서로 다른 단음절어표를 사용 하고 있다고 하였다(Byun et al., 2005). 또한 기존에 개발된 검 사어표는 일반 성인용으로 개발되어 유소아 및 아동을 대상으 로 시행하기 어렵고 육성으로만 검사를 진행해야 해서 기관 간 결과를 비교하기 어렵다는 단점이 있다. 이러한 문제점을 고려 하여 2010년에 다양한 연령대를 대상으로 CD 음원을 통해 이 음절, 단음절, 문장인지도 검사가 가능한 한국어 어음청각검사 도구가 제작되었다(Lee et al., 2010).

위에서 설명한 검사 도구들은 모두 소음이 없는 조용한 듣기 환경에서 어음 인지 능력을 평가하도록 개발된 도구들이다. 조 용한 듣기 환경에서 시행한 어음청각검사 결과는 가청 정도에 따른 영향을 확인하거나 순음청력검사 결과의 신뢰도를 확인 할 때, 그리고 보청기 혹은 인공와우 착용 대상자를 선정할 때 유용하게 사용할 수 있다. 그러나 현대인들은 다양한 소음 속 에서 일상생활을 하고 있으므로 조용한 환경에서 단어만을 제 시하는 검사 결과로 소음이 존재하는 일상생활 속 의사소통 능 력을 판단하는 데 한계가 있어(Killion \& Niquette, 2000; Killion et al., 2004; Kollmeier et al., 2015; Nilsson et al., 1994), 미 국, 영국 등의 의료 선진국에서는 다양한 소음 하 문장인지 평 가 도구를 개발하였고 검사를 위한 표준화 작업을 시행하였다.

현재까지 개발된 소음 하 문장인지 평가 도구는 목표 문장과 소음의 강도 레벨을 조절하는 방법에 따라 고정형(fixed) 혹은 변동형(adaptive) 검사로 구분한다. 고정형 소음 하 문장인지 검사에는 Speech Perception in Noise test (SPIN) (Kalikow et al, 1977), Connected Speech Test (CST) (Cox et al., 1987), City University of New York (CUNY) (Boothroyd et al., 1985) 등 이 있다. SPIN, CST, CUNY 검사 모두 1970 1980년대에 고안 된 검사 도구로 하나의 고정된 신호대잡음비(signal-to-noise ratio, SNR)에서 문장을 제시하고 그 결과를 백분율로 산출한 다. SPIN 검사의 경우 다화자잡음(multitalker noise) 속에서 문 장 내 문맥적 단서로 목표 단어를 예측하기 쉬운(high-predictability, HP) 문장 25개, 예측하기 어려운(low-predictability,
LP) 문장 25 개로 구성되어 있다. SPIN 검사는 소음 하 문장인 지 능력을 백분율로 점수화하는 것 외에 $\mathrm{HP}$ 와 LP 목록 간 결 과 차이를 통해 청자가 문맥 단서를 얼마나 잘 활용하는 지를 파악하는 데 활용할 수 있다. CST 검사의 경우 보청기 혜택을 측정하기 위한 목적으로 개발되었다. 검사 시작 전 청자에게 각 구문의 주제를 말해준 후, 청자에게 다화자잡음 속에서 10 개의 문장으로 이루어진 구문을 제시하여 청자가 각 구문에 포함된 25 개의 중심 단어를 얼마나 정확하게 인지하였는지 백분율로 점수화한다. CUNY 검사의 경우 조용한 상황 $(65 \mathrm{dBA})$ 혹은 다 화자잡음을 이용한 소음 하 상황(10 dB SNR)에서 3 14개의 단 어를 포함하는 문장을 제시하여 검사를 시행한다. CUNY 검사 도구는 12 개의 문장으로 구성된 72 개의 목록을 포함하므로 다 른 검사 도구에 비해 문장 목록의 수가 많으며, 채점을 위해 목 록 내 총 102 개의 단어에 대한 대답을 기준으로 백분율로 점수 화한다.

위에 나열한 고정형 소음 하 문장인지 검사 도구 모두 백분율 로 검사 결과를 산출하므로 난청인 상담 시 정보를 전달할 때 유용하게 사용할 수 있다. 그러나 고정형 소음 하 문장검사의 경 우 고정된 SNR 조건을 사용해야 하므로 어떤 난청인에게는 너 무 쉬워 천장효과(ceiling effect)를 보이거나 어떤 대상자에게는 너무 어려워 바닥효과(floor effect)를 보일 수 있어 인지도 변화 를 적절하게 평가하는 것이 어려울 수 있다(Vaillancourt et al., 2005). 이러한 문제점을 극복하기 위해 여러 SNR에서 고정형 소음 하 검사를 시행할 경우 검사 시간이 오래 걸린다는 또 다 른 임상적 한계에 부딪치게 된다. 이 외에 결과 산출을 위해 검 사자가 직접 백분율로 계산하는 등 부수적인 수고도 요구된다.

이러한 한계점을 고려하여 청자의 반응에 따라 자극음 혹은 소음의 강도를 변화하는 변동형(adaptive) 소음 하 문장인지 검사 도구가 다수 개발되었다. 변동형 소음 하 문장인지 검사에 서는 대상자가 충분히 들을 수 있는 SNR에서 문장을 제시하 기 시작하여 대상자가 정반응을 보이면 SNR을 낮추고 오반응 을 보이면 SNR을 높이는 방법으로 검사를 진행한다. 변동형 검사의 경우 어떠한 알고리즘을 통해 SNR이 변동되는지, 대상 자의 소음 하 인지도 결과를 산출하고 판독하는 방법 등에 있 어서 서로 다르다. Soli \& Wong(2008)은 소음 하 어음인지 검 사 시 자극음 및 소음의 특성, 검사 환경, 대상자, 검사 절차 등 이 검사 결과에 영향을 주므로 검사 도구의 개발 시 이러한 요 소들을 이해하는 것이 중요하다고 하였다. 따라서 본 종설에서 는 국외 임상 및 연구 분야에서 널리 사용되고 있는 변동형 소 음 하 문장인지 검사 도구의 특성을 파악하여 1) 각 검사 도구 에서 목표 문장과 배경 소음을 어떻게 구성하였고, 2) 어떠한 방 법으로 변동형 검사를 시행하며 점수화하는지, 3) 결과 판독을 위한 규준 및 판독 방법의 차이를 비교 분석하였다. 이와 같이 
각 도구의 특성, 장단점 등을 이해하여 향후 한국어판 변동형 소음 하 문장인지 검사 도구의 개발에 기초 자료로 사용하고자 한다.

\section{REVIEW OF ADAPTIVE SENTENCE-IN- NOISE TESTS}

현재 미국 및 유럽 등의 의료 선진국에서 다양한 변동형 소 음 하 문장인지 검사 도구를 개발하여 임상 및 연구의 목적으 로 널리 사용하고 있다[Hearing in Noise Test (HINT), Nilsson et al., 1994; Quick Speech-in-Noise test (QuickSIN ${ }^{\mathrm{TM}}$ ), Etymotic Research, 2001, Killion et al., 2004; Matrix test, Akeroyd et al., 2015]. 각 검사 도구에서 사용한 목표 문장과 배경 소음의 구성, 검사 절차 및 점수화 방법, 검사의 규준화 및 결 과 판독 방법의 순서로 각 검사 도구의 특성을 정리하였다.

\section{Sources of target sentences and background noise}

소음 하 문장인지 검사에서는 소음 속에서 문장을 제시하고 문장 전체 혹은 문장 내 중심 단어를 얼마나 정확하게 인지하 는지를 평가한다. 자극음으로 사용하는 문장과 배경 소음의 특 성이 검사 결과에 상당한 영향을 미치므로 도구 개발 시 문장 의 추출 및 구성, 소음 제작 등에 신중을 가해야 한다. 대표적으 로 많이 사용되는 변동형 소음 하 문장인지 검사 도구인 HINT (Nilsson et al., 1994), QuickSIN (Killion et al., 2004), Matrix (Wagener et al., 1999a, 1999b, 1999c)에서 어떻게 목표 문장과 소음을 구성하였는지 자세한 정보를 Table 1에 정리하였다.

미국식 영어판 HINT 검사에 대해 살펴보면(Nilsson et al., 1994) 문장을 새로 발췌하지 않고 기존의 영국식 영어 문장인 BKB (Bench et al., 1979) 문장을 미국식 영어로 수정하여 HINT 검사용 문장을 1차 추출하였다. BKB 문장은 8 15세 영 국 초등학생의 표현 어휘를 위주로 추출한 간단하고 길이가 짧 은 문장이다. 이와 같이 저학년 초등학생이 읽기 가능한 일상 대화 속 짧고 쉬운 문장을 활용한 이유는 문장 내 단어의 어휘 적(lexical) 특성, 문장의 문법적 복잡성, 문장 길이 등의 요소 가 대상자의 어음 인지에 최대한 영향을 미치지 않게 하기 위 해서이다. 1차로 추출된 문장 후보 중 자연성(naturalness) 검 사에서 통과한 문장만을 목표 문장으로 포함하였다. 최종 250 개의 문장(총 25 개 목록, 목록당 10 개의 문장)을 남성 화자가 녹음한 후 음원을 디지털로 변환하였고 각 음원의 실효값(root mean square)을 동일하도록 조절한 후 최종 음원의 음질, 명료 도 등을 확인하였다. Table 1에 제시하였듯이, HINT 검사에서 는 어음 스펙트럼을 가진 비유동 소음(stationary speech spectrum noise)을 사용한다. 비유동 소음은 시간에 따라 변동적 특
성을 보이지 않으므로 여러 시간 구간마다 동일한 스펙트럼을 가 진다. 1 2명의 화자가 이야기하는 배경어음(competing speech) 을 사용할 경우 자연스러운 의사소통 상황을 보다 잘 반영할 수 있어 검사 도구의 생태학적 타당도(ecological validity) 면에 서 더 우수할 수 있다. 그러나 1 2명 화자의 배경어음을 제시할 경우 음향적 차폐(energetic masking)와 의미적 차폐(informational masking)의 영향이 모두 존재하므로 두 가지 차폐의 영 향을 구분하기 어렵다. HINT 검사는 청자의 청력이 소음 하 어음인지에 미치는 영향을 평가하는 데 주 목적을 두므로 상위 단계에 속하는 의미적 차폐의 영향이나 인지 능력 및 기억력 등 의 영향을 최소화하고자 비유동소음을 제작하여 제시하였다. HINT 검사는 미국식 영어 외에(Nilsson et al., 1994) 15개의 언 어로 개발되어 있다(Table 1). 이 중 한국어판 HINT 검사 도구 는 2008년에 발표되었는데(Moon et al., 2008) 개발 후 검사를 위해 표준화가 일부 진행되었다. 한국어판 HINT 검사 도구는 한 국어로 개발된 유일한 변동형 소음 하 문장인지 검사 도구이나, 일반적인 컴퓨터가 아닌 고가의 장비(HINT pro, Bio-logic ${ }^{\circledR}$ sys., Mundelein, IL, USA)만을 통해서만 검사가 가능하여 국내 소규모 임상 현장에서 활용하기 어렵고 현재 한국어판 HINT 검사 도구의 판매가 중지되어 더 이상 구입이 불가능하다.

2004년에 발표된 Quick Speech-in-Noise Test (QuickSIN ${ }^{\mathrm{TM}}$ ) 검사는 Speech-in-Noise ( $\mathrm{SIN}^{\mathrm{TM}}$, Elk Grove Village, IL, USA) (Etymotic Research, 1993) 도구를 수정하여 보다 쉽고 빠르게 검사를 시행할 수 있도록 보완한 것이다(Killion et al., 2004). QuickSIN 검사에서는 4명 화자의 배경 어음(competing speech) 속에서 여성 화자가 녹음한 Institute of Electrical and Electronics Engineers (IEEE) 문장을 목표 문장으로 제시한다. IEEE 문장은 비교적 문맥적 단서가 많지 않은 것이 특징이며, Killion et al.(2004)은 최종 QuickSIN 문장 목록을 추출하기 위해 25명의 건청인에게 여러 저주파수통과(low-pass) 필터 조 건에서 다양한 목록 내 문장을 제시하여 평균 값에서 $\pm 2.2 \mathrm{~dB}$ 이내의 결과를 보이는 목록을 최종 추출하였다. 이러한 과정을 통해 리스트 간 동질성이 확보된 12 개의 기본검사 목록(목록 1 12), 연구용으로 추가한 3쌍의 문장 목록(목록 13/14, 15/16, 17/18), 연습용 목록 3개(목록 A, B, C)를 최종 목록으로 포함 하고 있다. 그 외에도 방향성 마이크로폰의 효과를 평가하기 위한 목록(목록 1 12와 다화자잡음을 서로 다른 채널에 녹음), 급경사형(ski-slope) 난청인을 위한 목록(목록 1 12, 13/14, $15 / 16$ 에서 고주파수만 $30 \mathrm{~dB}$ 증가한 목록), 보청기 효과를 평 가하기 위한 목록 등으로 사용할 수 있도록 하였다. QuickSIN 목록은 6개의 문장으로 구성되어 있고 각 문장은 목표 단어 5 개를 포함한다. 채점 시 5 개의 중심 단어를 얼마나 옳게 인지하 였는지를 통해 $50 \%$ 인지도를 보이는 SNR을 찾는다. 
HINT 검사와 QuickSIN 검사 모두 소음이 있는 일상생활 속에서 청자가 얼마나 듣기 어려운지를 평가하는 것이 목적이 다. 청자가 일상적으로 비유동 소음뿐 아니라 여러 화자가 이야 기하는 유동적(fluctuating) 소음 또한 자주 접하게 되므로 QuickSIN 검사 도구의 개발자들은 검사 도구의 안면타당도 (face validity)를 높이고자 실제 의사소통에서 자주 접하는 배 경 소음을 검사에 사용하고자 하였다. 보통 3 4명이 같이 대화 하는 상황에서 이야기 하는 경우가 많다는 점을 고려하여 총 4
명의 화자(남성 1 명, 여성 3명)가 동시에 이야기하는 다화자잡 음을 QuickSIN 검사 도구의 배경 소음으로 사용하였다.

Matrix 소음 하 문장인지 검사 도구는 Hagerman(1982)이 개 발한 스웨덴어 버전 객관식 문장에 기반하여 다양한 언어 버전 으로 검사 도구가 확장되었다. Matrix 검사에서는 10 개의 행, 5 개의 열로 이루어진 $10 \times 5$ 행렬(Matrix)에 포함된 단어의 조 합으로 생성 가능한 문장을 사용하며, 각 문장은 주어, 동사, 수사, 형용사, 목적어에 해당하는 단어들의 조합으로 이루어져

Table 1. A comparison of target sentences and background noise among the HINT, the QuickSIN ${ }^{\top M}$, and the matrix sentence test

\begin{tabular}{|c|c|c|c|c|c|}
\hline Test & $\begin{array}{c}\text { Sources of target } \\
\text { sentences }\end{array}$ & $\begin{array}{c}\text { Total number } \\
\text { of sentences } \\
\text { and lists }\end{array}$ & $\begin{array}{l}\text { Gender } \\
\text { of talker }\end{array}$ & Type of noise & $\begin{array}{l}\text { Other multilingual versions } \\
\text { (sorted by published year) }\end{array}$ \\
\hline $\begin{array}{l}\text { HINT } \\
\text { (Nilsson et } \\
\text { al., 1994) }\end{array}$ & $\begin{array}{l}\text { Adaptation of } \\
\text { existing } \\
\text { Bamford- } \\
\text { Kowal-Bench } \\
\text { sentences }\end{array}$ & $\begin{array}{l}250 \text { sentences } \\
\text { for } 25 \text { lists } \\
\text { (10 sentences } \\
\text { per list) }\end{array}$ & Male & $\begin{array}{l}\text { Stationary speech- } \\
\text { shaped noise whose } \\
\text { long-term spectrum is } \\
\text { matched to the } \\
\text { long-term spectrum } \\
\text { of speech material }\end{array}$ & $\begin{array}{l}\text { Canadian French HINT (Vaillancourt et al., 2005, } \\
\text { 2008), Cantonese HINT (Wong \& Soli, 2005; } \\
\text { Wong, 2008), Swedish HINT (Hällgren et al., } \\
\text { 2006), Mainland Mandarin HINT (Wong et al., } \\
\text { 2007), Brazilian Portuguese HINT (Bevilacqua } \\
\text { et al., 2008), Bulgarian HINT (Lolov et al., 2008), } \\
\text { Castilian Spanish HINT (Huarte, 2008), } \\
\text { Japanese HINT (Shiroma et al., 2008), Korean } \\
\text { HINT (Moon et al., 2008), Latin American } \\
\text { Spanish (Baron de Otero et al., 2008), } \\
\text { Malay HINT (Kar Quar et al., 2008), Norwegian } \\
\text { HINT (Myhrum \& Moen, 2008), Taiwanese } \\
\text { Mandarin HINT (Wong \& Huang, 2008), } \\
\text { Turkish HINT (Cekic \& Sennaroglu, 2008), } \\
\text { Danish HINT (Nielsen \& Dau, 2011) }\end{array}$ \\
\hline $\begin{array}{l}\text { QuickSIN }^{\mathrm{TM}} \\
\text { (Killion et } \\
\text { al., 2004) }\end{array}$ & $\begin{array}{l}\text { Adaptation } \\
\text { (shortening) } \\
\text { of existing } \\
\text { SIN }^{\mathrm{TM}} \\
\text { test (IEEE } \\
\text { sentences) }\end{array}$ & $\begin{array}{l}108 \text { sentences } \\
\text { for } 18 \text { lists } \\
\text { ( } 12 \text { lists for } \\
\text { basic test, } 6 \\
\text { additional } \\
\text { lists for } \\
\text { research) } \\
\text { (6 sentence per } \\
\text { list) }\end{array}$ & Female & $\begin{array}{l}\text { Four-talker competing } \\
\text { speech noise }\end{array}$ & \\
\hline $\begin{array}{l}\text { Matrix } \\
\text { Sentence } \\
\text { Test } \\
\text { (Wagener } \\
\text { et al., } \\
\text { 1999a, } \\
\text { 1999b, } \\
\text { 1999c) }\end{array}$ & $\begin{array}{l}\text { Creation of a } \\
\text { new sentence } \\
\text { test based } \\
\text { on a } 5 \times 10 \\
\text { word } \\
\text { matrix with } \\
\text { columns } \\
\text { containing ten } \\
\text { names, verbs, } \\
\text { numbers, } \\
\text { adjectives and } \\
\text { objects }\end{array}$ & $\begin{array}{l}200 \text { sentences } \\
\text { for } 10 \text { lists ( } 20 \\
\text { sentences per } \\
\text { list) }\end{array}$ & Male & $\begin{array}{l}\text { Stationary speech- } \\
\text { shaped noise whose } \\
\text { long-term spectrum is } \\
\text { matched to the long- } \\
\text { term spectrum } \\
\text { of speech material }\end{array}$ & $\begin{array}{l}\text { Danish (Wagener, 2003), Swedish (Wagener, } \\
\text { 2004), British English (Hewitt, 2007), Norwegian } \\
\text { (Øygarden, 2009), Polish (Ozimek et al., 2010), } \\
\text { Spanish (Hochmuth et al., 2012), French (Jansen } \\
\text { et al., 2012), American English (Zokoll et al., } \\
\text { 2013), Dutch (Houben et al., 2014), Finnish } \\
\text { (Dietz et al., 2014), Russian (Warzybok et al., } \\
\text { 2015), Italian (Puglisi et al., 2015), Turkish } \\
\text { (Zokoll et al., 2015), Australian English } \\
\text { (Kelly et al., 2016) }\end{array}$ \\
\hline
\end{tabular}

SNR-50: signal-to-noise ratio where correct sentence recognition of 50\% occurs, SIN $^{\mathrm{TM}}$ : Speech-in-Noise, IEEE: Institute of Electrical and Electronics Engineers, HINT: Hearing in Noise Test, QuickSIN ${ }^{\mathrm{TM}}$ : Quick Speech-in-Noise Test 
있다. 문장 내 단어 선택 시 해당 언어의 음소 빈도 및 분포도, 어휘의 친숙도, 사용 빈도, 자연성, 중립성, 이름(주어)의 선호 도 등을 고려하여 선정하며, 문장의 자연성, 난이도, 타당도, 적 절성 등을 평가한 후 문장 음원을 녹음한다. Matrix 문장 검사 에서는 목표 화자의 어음의 스펙트럼을 배경 소음에 최대한 반 영하기 위해 목표 화자가 발화한 문장 여러 개를 선택한 후, 각 문장의 시작점을 달리한 채 최소 30 회 이상 겹치게 하여(superimpose) 일반 어음이 아닌 해당 목표 화자가 발화한 문장의 장기평균스펙트럼을 가지는 소음을 배경 소음으로 사용한다.

앞서 소개한 HINT와 QuickSIN 검사 도구는 대상자가 문장 을 듣고 들은 대로 따라 말해야 하는 보기가 없는(open-set) 형식의 검사이다. Table 1 에 제시하였듯이 HINT 검사 도구에는 총 250개의 문장이, QuickSIN 검사 도구에는 총 108개의 문 장이 포함되어 있다. 이와 같이 한정된 개수의 문장을 검사에 반복적으로 사용할 경우 대상자가 문장을 외우거나 학습효과 가 발생할 수 있다. Matrix 검사 도구에서는 5개의 단어(주어, 동사, 수사, 형용사, 목적어)당 10 개의 보기가 존재하며 $10 \times 5$ 행렬(Matrix)에 포함된 단어의 조합으로 총 $10^{5}$ 개의 문장을 생 성하여 사용할 수 있으므로 학습효과에 대한 우려를 최소화하 고 반복적으로 검사를 시행하는데 유리하다. 또한 Matrix 검사 는 대상자가 직접 컴퓨터를 활용하여 들은 단어를 직접 선택하 게 할 수 있어 문장의 제시 속도를 본인이 조절할 수 있고 검사 자의 수고가 덜 요구된다는 장점이 있다.

Table 1에 나열하였듯이 현재 14개의 언어 버전으로 Matrix 소음 하 문장인지 검사 도구가 개발되었고, 이 외에 한국어, 아 랍어, 페르시아어, 일본어, 중국어, 히브리어, 힌두어 등의 언어로 도 개발 진행 중이다(Yi, 2006). 모든 다국어판 Matrix 검사 도구 에서 $10 \times 5$ 행렬(Matrix)을 사용하며 유사한 방식으로 문장을 선정하므로 문장의 복합성 혹은 문맥적 단서의 영향을 최소화 할 수 있다. 특히 외국인 대상자에게 검사를 진행할 때 $10 \times 5$ 행렬(Matrix)을 통한 객관식 검사가 가능하므로 대상자의 모국 어를 몰라도 검사를 진행할 수 있어 임상적 활용도를 극대화할 수 있다.

\section{Test procedure and scoring formula}

변동형 소음 하 문장인지 검사에서는 어떻게 목표 문장과 배 경 소음을 제시하며, 변동형 검사를 진행하기 위해 몇 $\mathrm{dB}$ 단위 로 어떻게 SNR을 조절하는지, 어떤 듣기 환경에서 검사를 진행 할 수 있으며, 어떤 방법을 통해 점수화 하는지에 따라 검사 결 과가 상이할 수 있다. 따라서 HINT, QuickSIN, Matrix 검사 도 구 각각에서 위의 다양한 요소들이 어떻게 다른지를 비교하기 위해 Table 2에 이를 정리하였다.

$\mathrm{HINT}$ 검사에서는 소음의 강도를 항상 $65 \mathrm{~dB}(\mathrm{~A})$ 에서 제시
하며 대상자의 반응에 따라 목표 문장의 강도를 올리거나 내린 다. 조용한 상황과 소음이 있는 조건에서 문장을 제시하여 청 자의 소음 하 문장인지 능력을 SNR로 산출한다. 먼저 소음이 없는 조용한 상황에서의 검사를 진행하며, 이때 $20 \mathrm{~dB}(\mathrm{~A})$ 에서 문장을 제시하여 대상자의 대답에 따라 $4 \mathrm{~dB}$ 간격으로 문장의 강도를 조절한다[단위: $\mathrm{dB}(\mathrm{A})]$. 그 다음 순서로 소음이 있는 조 건에서 소음 하 문장인지 능력을 측정하며 $50 \%$ 의 인지도를 보 이는 SNR 지점을 찾는다. HINT 검사에서 구한 문장인지역치 를 reception threshold for sentences (RTS)라 표현하며 $\mathrm{dB}$ $\mathrm{SNR}$ 의 단위로 결과를 기록한다.

HINT 검사에서는 검사를 실시하기 전 4개의 연습용 문장 을 이용하여 친숙화를 거친 후 20 개의 문장을 검사용 문장으 로 제시한다. 검사 시 협차법(bracketing method)을 이용하여 검사가 너무 쉽거나 너무 어렵지 않도록 한다. 20 개의 문장 중 1 번 문장의 경우 문장 내 단어를 모두 맞출 때까지 $4 \mathrm{~dB}$ 간격 으로 문장의 강도를 증가한다(ascending). 만약 1번 문장을 옳 게 맞춘 레벨이 $52 \mathrm{~dB}(\mathrm{~A})$ 이었다면 2 4번 문장을 제시하고 대 상자의 대답에 따라 $4 \mathrm{~dB}$ 간격으로 문장의 강도를 변화시킨다 (맞추면 내리고 틀리면 올림). 나머지 5 20번까지의 16 개 문장의 강도는 $2 \mathrm{~dB}$ 간격으로 조절한다. $5 \sim 20$ 번 문장의 RTS와 20 번 문 장에 대한 정반응/오반응 여부를 기준으로 한 21번 문장의 RTS 를 구하여 최종 RTS에 해당하는 역치를 구한다.

$\mathrm{HINT}$ 검사에서는 항상 대상자의 정면 $\left(0^{\circ}\right)$ 에서 목표 문장을 제시하며, 소음은 대상자를 기준으로 정면 $\left(0^{\circ}\right)$, 오른쪽 $\left(90^{\circ}\right)$, 왼쪽 $\left(270^{\circ}\right)$ 방향에 위치한 스피커 중 하나에서 제시한다. 대부분의 문장 인지 검사 도구에서는 문장 내 중심 단어를 몇 개 맞추었 는지를 점수화(keyword scoring)하여 결과를 판독하나, HINT 검사에서는 청자가 문장 내 단어를 옳게 따라하는 경우에만 정 반응을 보였다고 인정한다(영어에서 "a" 혹은 "the" 간 오류, "is"와 "was" 간 오류와 같은 미미한 실수는 제외). 즉, 문장 내 단어 한 개만 놓쳐도 오반응으로 평가하므로 SNR이나 검사 환 경 등의 변화에 보다 더 민감한 특징을 가진다.

QuickSIN 검사에서는 고정된 강도에서 목표 문장을 제시하 고 대상자의 반응에 따라 소음의 강도를 변화시킨다. HINT 검 사와 다르게 대상자의 청력에 따라 검사 시작 레벨이 다른데, 대상자의 평균 순음청력역치가 $45 \mathrm{~dB} \mathrm{HL}$ 이하라면 $70 \mathrm{~dB} \mathrm{HL}$ 에서 목표 문장을 제시하고, 평균순음청력역치가 $45 \mathrm{~dB} \mathrm{HL}$ 초 과라면 대상자가 크지만 듣기 좋다(“loud but ok”)고 답한 레벨 에서 문장을 제시한다.

QuickSIN 검사에서는 대상자의 반응에 상관없이 $25 \mathrm{~dB}$ $\mathrm{SNR}$ 에서 시작하여 $5 \mathrm{~dB}$ 간격으로 SNR을 감소시킨다(descending paradigm). 각 목록에 총 5개의 문장이 포함되어 있으므로 첫 번째 문장은 $25 \mathrm{~dB}$ SNR에서 마지막 여섯 번째 문장은 0 
Table 2. A comparison of test procedure and scoring information among the HINT, the QuickSIN ${ }^{\top M}$, and the Matrix Sentence test

\begin{tabular}{|c|c|c|c|c|c|c|c|}
\hline Test & $\begin{array}{c}\text { Presentation } \\
\text { level of sentence }\end{array}$ & $\begin{array}{l}\text { Presentation } \\
\text { level of noise }\end{array}$ & Step size & $\begin{array}{c}\text { Possible evaluation } \\
\text { environments }\end{array}$ & $\begin{array}{l}\text { Scoring } \\
\text { method }\end{array}$ & $\begin{array}{l}\text { What is measured as } \\
\text { score }\end{array}$ & $\begin{array}{c}\text { Administration } \\
\text { time }\end{array}$ \\
\hline $\begin{array}{l}\text { HINT } \\
\text { (Nilsson et } \\
\text { al., 1994) }\end{array}$ & $\begin{array}{l}\text { Bracketing } \\
\text { method is used } \\
\text { to determine } \\
\text { initial } \\
\text { presentation } \\
\text { level at \# } 1 \\
\text { sentence is } \\
\text { correctly } \\
\text { identified }\end{array}$ & $\begin{array}{l}\text { Fixed noise } \\
\text { level } \\
\text { (65 dBA) }\end{array}$ & $\begin{array}{l}4 \text { dB-step } \\
\text { change for } \\
\# 2-\# 4 \\
\text { sentences, } \\
2 \text { dB-step } \\
\text { change for } \\
\# 5-\# 20 \\
\text { sentences }\end{array}$ & $\begin{array}{l}\text { Sound-field test } \\
\text { (sentences from } \\
\text { front, noise from } \\
\text { front, left, or } \\
\text { right) } \\
\text { Possible with } \\
\text { headphone that } \\
\text { simulates sound- } \\
\text { field performance }\end{array}$ & $\begin{array}{l}\text { Whole } \\
\text { sentence scor- } \\
\text { ing (some } \\
\text { substitutions } \\
\text { are accept } \\
\text { able) }\end{array}$ & $\begin{array}{l}\text { Average Reception } \\
\text { Threshold for Speech } \\
\text { where correct } \\
\text { sentence recognition } \\
\text { of } 50 \% \text { occurs } \\
\text { [score in dB(A) for } \\
\text { quiet, score in dB } \\
\text { SNR for noise] }\end{array}$ & $\begin{array}{l}\text { About one } \\
\text { minute for a } \\
\text { single list }\end{array}$ \\
\hline $\begin{array}{l}\text { QuickSIN }^{\mathrm{TM}} \\
\text { (Killion et } \\
\text { al., 2004) }\end{array}$ & $\begin{array}{l}\text { Fixed level } \\
(70 \mathrm{~dB} H \mathrm{HL} \text { for } \\
\text { listeners with } \\
\mathrm{PTA} \leq 45 \mathrm{~dB} \\
\mathrm{HL} \text { as starting } \\
\text { presentation } \\
\text { level, “loud but } \\
\text { OK” level for } \\
\text { listeners with } \\
\text { PTA > } 45 \mathrm{~dB} \\
\text { HL) }\end{array}$ & $\begin{array}{l}\text { Level of } \\
\text { noise is } \\
\text { adaptively } \\
\text { decreased. }\end{array}$ & $\begin{array}{l}5 \text { dB-step } \\
\text { decrement } \\
\text { from } 25 \\
\text { dB SNR to } \\
0 \text { dB SNR }\end{array}$ & $\begin{array}{l}\text { Sound-field test } \\
\text { (both sentences } \\
\text { and noise from } \\
\text { front) } \\
\text { Possible with } \\
\text { insert earphones } \\
\text { or headphones } \\
\text { for binaural test }\end{array}$ & $\begin{array}{l}\text { Key word } \\
\text { scoring based } \\
\text { on } 5 \\
\text { keywords } \\
\text { in each } \\
\text { sentence }\end{array}$ & $\begin{array}{l}\text { SNR loss which is } \\
\text { defined as the } \\
\text { difference of signal- } \\
\text { to-noise ratio here } \\
\text { correct recognition } \\
\text { of } 50 \% \text { occurs of the } \\
\text { hearing-impaired } \\
\text { and normal-hearing } \\
\text { listeners }\end{array}$ & $\begin{array}{l}\text { About one } \\
\text { minute for a } \\
\text { single list }\end{array}$ \\
\hline $\begin{array}{l}\text { Matrix Sen- } \\
\text { tence Test } \\
\text { (Wagener } \\
\text { et al., } \\
\text { 1999a, } \\
\text { 1999b, } \\
\text { 1999c) }\end{array}$ & $\begin{array}{l}\text { Level of } \\
\text { sentence is } \\
\text { adaptively } \\
\text { changed ( } 0 \mathrm{~dB} \\
\text { SNR as } \\
\text { starting level) }\end{array}$ & $\begin{array}{l}\text { Fixed noise } \\
\text { level (65 } \\
\text { dB SPL) }\end{array}$ & $\begin{array}{l}\text { Step size } \\
\text { varied } \\
\text { based on } \\
\text { maximum } \\
\text { likelihood } \\
\text { estimator }\end{array}$ & $\begin{array}{l}\text { Monaural free- } \\
\text { field equalized } \\
\text { headphone. } \\
\text { Possible with } \\
\text { sound-field test } \\
\text { (both sentences } \\
\text { and noise from } \\
\text { front) }\end{array}$ & $\begin{array}{l}\text { Key word } \\
\text { scoring based } \\
\text { on } 5 \\
\text { keywords in } \\
\text { each sentence }\end{array}$ & $\begin{array}{l}\text { Sentence recognition } \\
\text { threshold where } \\
\text { correct sentence } \\
\text { recognition of } 50 \% \\
\text { occurs (score in dB } \\
\text { SNR) based on a } \\
\text { maximum likelihood } \\
\text { procedure (Brand \& } \\
\text { Kollmeier, 2002). }\end{array}$ & $\begin{array}{l}\text { About four } \\
\text { minute when } \\
\text { using } 20 \\
\text { sentences }\end{array}$ \\
\hline
\end{tabular}

HINT: Hearing in Noise Test, SNR: signal-to-noise ratio, QuickSIN ${ }^{\mathrm{TM}}$ : Quick Speech-in-Noise Test, PTA: puretone threshold average

$\mathrm{dB} \mathrm{SNR}$ 에서 제시하게 된다. 다른 검사와는 달리 수정상승법 을 이용하지 않고 각 $\mathrm{dB} \mathrm{SNR}$ 에서 문장 한 개씩 제시하게 되므 로 검사 소요시간이 매우 짧다는 특징을 가진다. QuickSIN 검 사 목록 한 개를 이용할 경우 검사를 진행하는 데 약 1 분이 소 요되며, 진단의 목적을 위해서는 검사를 2 회 시행한 후 결과를 평균하여 판독할 것을 권장한다(Killion et al., 2004). 또한 QuickSIN 검사는 음장뿐 아니라 헤드폰이나 삽입형 이어폰을 이용하여 진행할 수 있다.

QuickSIN 문장 검사에서는 대상자가 $50 \%$ 의 소음 하 문장인 지도를 보이는 SNR을 구할 때 Spearman-Kärber 공식(Finney, 1952)을 이용하며, 그 공식은 Eq. [1]과 같다.

$$
\begin{aligned}
& \mathrm{i}+1 / 2(\mathrm{~d})-(\mathrm{d})(\# \text { of correct sentences }) /(\mathrm{w})=\text { SNR for } 50 \% \text { [1] } \\
& \mathrm{i}=\text { initial presentation level in } \mathrm{dB} \text { SNR }
\end{aligned}
$$

$$
\begin{aligned}
& \mathrm{d}=\text { step size for level decrement } \\
& \mathrm{W}=\text { the number of words per decrement }
\end{aligned}
$$

QuickSIN 검사 도구는 목록당 30개의 중심단어를 포함하며 (목록 내 6개의 문장, 문장당 5 개의 중심 단어) 총 30 개의 중심 단어 중 몇 개를 옳게 맞추었는지를 계산하여 SNR을 구한다. 예를 들어 어떤 난청인이 총 30 개의 중심 단어 중 20 개를 맞추 었다면 위의 Spearman-Kärber 공식을 적용하였을 때 i = 25 (문장의 첫 제시레벨이 $25 \mathrm{~dB} \mathrm{SNR}), \mathrm{d}=5(5 \mathrm{~dB}$ 간격으로 강 도 조절), $\mathrm{w}=5$ 이다(문장당 5 개 중심 단어 포함). 이를 대입하 여 계산하면 대상자가 $50 \%$ 의 소음 하 문장인지도를 보인 SNR 은 $7.5 \mathrm{~dB}$ 이다 $(25+1 / 2(5)-5(20) /(5)=25+2.5-20=7.5)$.

QuickSIN 검사에서는 $50 \%$ 인지도에 해당하는 SNR을 구하 는 것에 그치지 않고 건청인이 $50 \%$ 인지도를 보이는 평균 SNR 수치와 난청인 대상자의 수치 차이를 비교하여 SNR loss를 구 
한다. 예를 들어 건청군이 $50 \%$ 인지도를 보이는 평균 수치가 2 $\mathrm{dB}$ SNR이고 난청인 대상자가 $7.5 \mathrm{~dB}$ SNR에서 $50 \%$ 의 인지도 를 보였다면, 그 난청인은 $5.5 \mathrm{~dB}$ SNR loss를 가진다고 해석한 다. 임상 현장에서 위의 계산을 하려면 시간이 많이 소요되므 로 이를 단순화하여 25.5에서 대상자가 맞춘 문장 내 단어의 개수를 빼 대상자의 SNR loss를 쉽게 구할 수 있게 하였다 (Killion et al., 2004). 따라서 대상자가 30개 중심 단어 중 20 개의 단어를 맞춘 경우 $5.5 \mathrm{~dB}$ SNR loss $(25.5-20=5.5)$ 를 가 짐을 쉽게 계산할 수 있다.

Matrix 검사에서는 HINT 검사와 유사하게 고정된 강도(65 $\mathrm{dB} \mathrm{SPL}$ )에서 소음을 제시하고 대상자의 대답에 따라 문장 강 도를 변동시킨다. QuickSIN 검사에서는 $5 \mathrm{~dB}$ 의 고정된 간격으 로만 레벨을 조절하였다면 Matrix 검사에서는 maximum likelihood estimator에 근거하여 SNR 단위를 변화시키며 $\mathrm{SNR}$ 을 조절한다. 음장으로 검사를 진행할 경우 대상자의 정면 에 위치한 스피커를 통해 문장을 제시하며, 헤드폰을 통해 한 귀에만 문장을 제시할 경우 free-field equalized headphone을 사용한다. Matrix 검사에서는 검사자가 강도를 직접 변화시키 지 않고 Oldenburg Measurement Applications (OMA) 소프트 웨어를 이용하여 자동으로 이를 조절한다. OMA 소프트웨어를 사용할 경우 $50 \%$ 에 해당하는 SNR 뿐 아니라 $20 \%, 80 \%$ 에 해 당하는 SNR도 쉽게 구할 수 있다. Matrix 검사에서 50\%의 인 지도를 보이는 SNR과 50\% 접점 기울기를 측정하기 위해 로지 스틱 모델 함수를 사용하였다. 그 공식은 Eq. [2]와 같다.

$$
\mathrm{SRT}_{\text {word }}(\mathrm{SNR})=\frac{1}{1+\exp \left[4 \mathrm{~S}_{50 \text { word }}\left(\mathrm{SRT}_{\text {word }}-\mathrm{SNR}\right)\right]}
$$

$\mathrm{SRT}_{\text {word }}=$ word$^{-}$-specific SRT in $\mathrm{dB}$ for $50 \%$ intelligibility $\mathrm{S}_{50 \text { word }}=$ slope at the SRT word

고정된 SNR을 사용하는 검사 도구와는 달리 SNR을 변화시 키는 변동형 소음 하 문장인지 검사에서는 다양한 난청 정도 를 가진 대상자에게 검사를 진행할 수 있어 난청인과 건청인의 데이터를 비교하는 데 더 용이하다(Soli \& Wong, 2008). 문장 의 강도는 고정시킨 채 소음의 레벨을 변화시키는 QuickSIN 검사와는 달리 HINT와 Matrix 검사에서는 소음의 레벨을 고 정한 채 대상자의 응답에 따라 문장 레벨을 조절하여 변동형 검사를 시행한다. 각 검사에서 서로 다른 방법으로 문장과 소 음을 제시하고 SNR을 조절하며 결과를 점수화하므로 각 검사 의 소요시간 및 평가 목적을 고려하여 검사 종류를 결정하는 것이 필요하겠다.

\section{Norms and interpretation of test results}

한 명의 대상자에게 어떠한 검사를 시행하고 그 검사 결과를 판독하기 위해서는 규준(norm) 집단의 점수와 비교하여 대상 자의 결과를 해석하고 상대적 위치를 파악할 수 있다. 규준 집 단이 모집단을 잘 대표하고 있어야 적절한 규준을 세울 수 있 는데, 청각학에서는 보통 건청인을 규준 집단으로 하여 점수의 분포를 확인한다. 각 검사 도구에서 검사 결과를 판독하기 위 해 규준 데이터를 구하는 것이 필수적이며, 집단 내 규준을 제 공하기 위해 보통 백분위 점수(percentile)를 이용하거나 표준 점수( $\mathrm{z}$ 점수), 표준 등급, 편차 등을 구한다. 이 중 백분위 점수 를 사용할 경우 계산이 쉽고 일반 사람들도 쉽게 이해할 수 있 다는 장점이 있다.

HINT 검사의 경우 소음 상황에서 50\%의 문장인지도를 보 이는 RTS 역치를 구한다. HINT 검사의 규준 표집에서는 건청 인의 RTS 평균 및 표준 편차 외에 특정 역치를 보이는 백분위 (percentile) 값에 해당하는 RTS를 함께 제시하였다. HINT 규 준 확립을 위해 대부분의 선행 연구에서 최소 16 명의 건청 성 인을 규준 집단의 대상자로 하였다 $(250 \sim 6,000 \mathrm{~Hz}$ 내 역치가 $25 \mathrm{~dB} \mathrm{HL}$ 이하). 예를 들어 미국 영어판 HINT 검사 도구의 경 우 44명의 건청인을 대상으로 규준 자료를 표집하였다(Nilsson et al., 1994). 13개의 언어로 개발된 HINT 규준 자료를 비교 분 석한 결과(Soli \& Wong, 2008), 조용한 상황에서 측정한 13개 언어의 평균 RTS는 $18.4 \mathrm{~dB}(\mathrm{~A})$ (표준편차: 3.5, 범위: 14.6 25.9) 였다. 소음이 정면에서 오는 조건에서의 평균 RTS는 $-3.9 \mathrm{~dB}$ $\mathrm{SNR}$ (표준편차: 0.8, 범위: -5.3 -2.6), 소음이 오른쪽에서 오는 조건에서의 평균 RTS는 $-11.2 \mathrm{~dB} \mathrm{SNR(표준편차:} \mathrm{0.9,} \mathrm{범위:}$ -12.4 -9.7), 소음이 왼쪽에서 오는 조건에서의 평균 RTS는 -11.3 dB SNR(표준편차: 0.9, 범위: -12.5 -10.1)이었다. 13개의 서로 다른 언어의 HINT 규준 자료를 살펴보면 수행-강도 함수 기울기가(performance-intensity function slope) 평균 $10 \% / \mathrm{dB}$ 에 매우 근접하였다(표준편차: 1.5). 이는 HINT 검사에서 SNR 을 $1 \mathrm{~dB}$ 변화하면 문장인지도가 약 $10 \%$ 의 변화를 보일 것을 의미한다(Soli \& Wong, 2008). 위에서 언급한 HINT 규준 자 료는 헤드폰을 사용하였을 경우의 자료이며, 각 언어의 규준 자료는 HINT 검사를 위한 소프트웨어에 내장되어 있어 검사 자가 임의로 바꿀 수 없다. 만약 음장 검사를 위한 HINT 규준 이 필요하다면 검사자가 실제 검사환경에서 수치를 직접 구하 여 HINT 데이터베이스에 이를 추가해야만 한다.

QuickSIN 검사 도구 개발 시 26명의 건청인을 대상으로 규 준 자료를 표집하였다(Killion et al., 2004). 앞서 설명하였듯이 QuickSIN 검사에서는 건청인과 난청인이 $50 \%$ 의 인지도를 보 이는 SNR을 계산하여 난청인의 SNR loss의 정도를 산출한다. 여기서 SNR loss 값이 클수록 건청인에 비해 해당 대상자가 소 
음 속에서 의사소통할 때 상대적으로 더 어려움을 의미한다. 만 약 0 3 dB의 SNR loss를 보이면 normal/near normal으로 건 청인보다 소음 하 인지 능력이 더 좋을 수 있다고 판독한다. 3 $7 \mathrm{~dB}$ 의 SNR loss를 보이면 mild SNR loss라 분류하며 배경소 음 속에서 건청인이 보이는 어려움 정도만 보일 것으로 해석한 다. 7 15 dB SNR loss를 보였다면 moderate SNR loss로 방향 성 마이크로폰을 적절하게 사용하는 것이 도움이 될 것으로 판독한다. $15 \mathrm{~dB}$ 을 초과한 severe SNR loss를 보였다면 보청기 증폭만으로는 어려움이 있어 $\mathrm{FM}$ 시스템과 같은 기타 보조기기 의 사용을 통해 SNR을 최대한 향상시켜야 한다고 해석한다 (Killion et al., 2004). 즉 QuickSIN 검사 결과로 단순히 대상자 의 소음 하 의사소통의 어려움만 확인하는 것이 아니라 대상자 에게 어떠한 보장구의 기능을 선택하는 것이 좋을지 상담 자료 로 활용할 수 있다는 장점이 있다.

2010년 이전에 6개의 언어로 Matrix 검사 도구가 개발되었고 (노르웨이어, 덴마크어, 독일어, 스웨덴어, 영국식 영어, 폴란드 어) 2010년부터 2016년 현재까지 9개의 언어로 Matrix 검사 도 구가 발표되었다(네덜란드어, 러시아어, 미국 영어, 스페인어, 이 탈리아어, 프랑스어, 핀란드어, 터키어, 호주 영어). 그 외에 한국 어, 이스라엘어, 이란어 등의 도구가 개발 진행 중이다. 14 개 국 어의 Matrix 소음 하 문장인지 검사 결과를 비교한 결과 최소 $-10 \mathrm{~dB}$ SNR에서 최대 $-6 \mathrm{~dB} \mathrm{SNR}$ 에서 $50 \%$ 의 문장인지도를 보였고 표준 편차가 0.9 이하였다(Kollmeier et al., 2015). 50\% 의 인지도를 접점으로 한 기울기를 비교한 결과 $10.2 \% / \mathrm{dB}$ 에서 $17.1 \% / \mathrm{dB}$ 범위에 속하였다. 언어에 상관없이 결과에서 SRT와 기울기가 매우 유사하였던 이유는 Matrix 검사 도구 개발 시 목록 간 동질성을 확보하는 최적화(optimization) 과정을 시행 하였기 때문이다. 다른 소음 하 문장인지 검사에서는 문장 간, 목록 간 심리측정함수(psychometric function)를 비교하였으나 Matrix 검사 도구에서는 SNR에 따라 단어별 인지도 점수를 확 인하고 동질하지 않은 단어의 음원 레벨을 수정하거나 버리는 (discarded) 등의 다양한 과정을 거치므로 서로 다른 언어임에 도 공통적으로 비교적 낮은 역치와 높은 기울기를 보였을 것으 로 추정하였다. Matrix 검사에서는 동일한 문법 구조를 가진 문 장을 반복하여 듣고 인지하는 검사이다. Matrix 검사에서 훈련 효과(training effect)의 영향을 확인하기 위해 $1 \mathrm{~dB}$ 간격으로 20 개의 Matrix 문장을 통해 검사를 약 5 회 시행한 결과 첫 번째와 두 번째 검사 결과에는 큰 차이가 났으나 그 이후로는 검사 결과 가 크게 다르지 않았다고 하였다(Hochmuth et al., 2012; Puglisi et al., 2015; Warzybok et al., 2015). 따라서 Matrix 검사에 서 훈련 효과를 최소화하기 위해 2개의 문장 목록을 통해 친 숙화 과정을 거친 후 실제 검사를 진행할 것을 권장한다.

소음 하 문장인지 검사는 검사의 목적 상 난청인을 대상으로
여러 번 검사를 시행하여 의사소통 능력의 변화를 관찰하는 경우가 많다. 따라서 검사-재검사 간 결과의 일치 정도, 목록 간 난이도의 유사 정도, 검사자 간 결과의 차이, 기존 검사와 신규 검사와의 상관성 등의 다양한 요소를 확인하여 도구의 신 뢰도를 확인하는 것이 중요하다. 또한 검사 도구가 실제로 측정 하고자 하는 것을 제대로 잘 측정하고 있는지, 얼마나 검사 도 구가 자연스러운 검사 환경을 반영하는지 등을 확인하기 위해 검사 도구의 타당도를 검증하는 것이 필요하다. 소음 하 Matrix 문장인지 검사의 경우 이와 같은 신뢰도, 타당도 검증이 필수적 이라고 보고하였으므로 향후 한국어 Matrix 검사 도구가 개발 된다면 간청인과 난청인 모두를 대상으로 체계적인 후속 연구 가 뒷받침되어야 하겠다.

\section{CONCLUSION}

조용한 듣기 상황에서 측정한 어음인지도 결과로는 소음이 있는 일상생활 속 의사소통 능력을 평가하거나 의사소통 장애 의 정도를 예측하는 데 한계가 있다. 국외에는 표준화 된 변동 형 소음 하 문장인지 검사 도구가 다양하여 임상 및 연구의 목 적에 따라 선택하여 사용할 수 있으나 국내의 경우 한국어로 표준화된 변동형 소음 하 문장인지 검사 도구가 부족하다. 현재 국외에서 활발히 사용되고 있는 검사 도구의 특성 및 장단점을 파악하여 향후 신뢰도와 타당도가 우수한 한국어검사 도구를 개발에 활용하는 것이 필요하겠다. 소음 하 문장인지 결과는 청 자의 의사소통 능력을 측정하는 목적 외에 난청인에게 적절한 청각재활을 계획하거나 상담 시에도 사용하여 임상에 보다 적극 적으로 활용할 필요가 있겠다.

중심 단어 : 변동형 소음 하 문장인지 평가 - 소음 하 어음 인지 어음청각검사.

\section{Acknowledgments}

이 논문은 2016년 대한민국 교육부와 한국연구재단의 지원을 받 아 수행된 연구입니다(NRF-2016S1A5A8020353). 본 연구에 참 여해 주신 난청인분들께 감사드립니다.

\section{REFERENCES}

Akeroyd, M. A., Arlinger, S., Bentler, R. A., Boothroyd, A., Dillier, N., Dreschler, W. A., et al. (2015). International Collegium of Rehabilitative Audiology (ICRA) recommendations for the construction of multilingual speech tests: ICRA Working Group on Multilingual Speech Tests. International Journal of Audiology, 54(Suppl 2), 17-22.

Baron de Otero, C., Brik, G., Flores, L., Ortiz, S., \& Abdala, C. (2008). The Latin American Spanish hearing in noise test. International Journal of Audiology, 47(6), 362-363.

Bench, J., Kowal, Å., \& Bamford, J. (1979). The BKB (Bamford-KowalBench) sentence lists for partially-hearing children. British Journal of Audiology, 13(3), 108-112. 
Bevilacqua, M. C., Banhara, M. R., Da Costa, E. A., Vignoly, A. B., \& Alvarenga, K. F. (2008). The Brazilian Portuguese hearing in noise test. International Journal of Audiology, 47(6), 364-365.

Boothroyd, A., Hanin, L., \& Hnath, T. (1985). A sentence test of speech perception: Reliability, set equivalence, and short term learning (Internal Report RCI 10). New York, NY: City University of New York.

Brand, T. \& Kollmeier, B. (2002). Efficient adaptive procedures for threshold and concurrent slope estimates for psychophysics and speech intelligibility tests. The Journal of the Acoustical Society of America, 111(6), 28012810.

Byun, S. W., Chung, S. M., Kim, H. S., \& Go, Y. M. (2005). A survey of phonetically balanced words lists used in training hospitals in Korea. Korean Journal of Otolaryngology-Head and Neck Surgery, 48(9), 10861090.

Campbell, G. A. (1910). XII. Telephonic intelligibility. The London, Edinburgh, and Dublin Philosophical Magazine and Journal of Science, 19(109), 152-159.

Cekic, S. \& Sennaroglu, G. (2008). The Turkish hearing in noise test. International Journal of Audiology, 47(6), 366-368.

Cox, R. M., Alexander, G. C., \& Gilmore, C. (1987). Development of the Connected Speech Test (CST). Ear and Hearing, 8(5 Suppl), 119S-126S.

Dietz, A., Buschermöhle, M., Aarnisalo, A. A., Vanhanen, A., Hyyrynen, T., Aaltonen, O., et al. (2014). The development and evaluation of the Finnish matrix sentence test for speech intelligibility assessment. Acta Oto-laryngologica, 134(7), 728-737.

Egan, J. P. (1948). Articulation testing methods. The Laryngoscope, 58(9), 955-991.

Etymotic Research (1993). The SIN Test (Compact Disk) 61 Martin Lane, Elk Grove Village, Illinois 60007.

Finney, D. J. (1952). Statistical method in biological assay (2nd ed.). London: Hafner.

Hagerman, B. (1982). Sentences for testing speech intelligibility in noise. Scandinavian Audiology, 11(2), 79-87.

Hahm, T. Y. (1962). Articulation function on the Korean speech in patients with hearing impairment. Catholic Medical College Journal, 5, 31-38.

Hällgren, M., Larsby, B., \& Arlinger, S. (2006). A Swedish version of the Hearing In Noise Test (HINT) for measurement of speech recognition. International Journal of Audiology, 45(4), 227-237.

Hewitt D. R. (2007). Evaluation of an English speech-in-noise audiometry test (unpublished master's thesis). University of Southampton, UK.

Hirsh, I. J., Davis, H., Silverman, S. R., Reynolds, E. G., Eldert, E., \& Benson, R. W. (1952). Development of materials for speech audiometry. Journal of Speech and Hearing Disorders, 17(3), 321-337.

Hochmuth, S., Brand, T., Zokoll, M. A., Castro, F. Z., Wardenga, N., \& Kollmeier, B. (2012). A Spanish matrix sentence test for assessing speech reception thresholds in noise. International Journal of Audiology, 51(7), 536-544.

Houben, R., Koopman, J., Luts, H., Wagener, K. C., Van Wieringen, A., Verschuure, H., et al. (2014). Development of a Dutch matrix sentence test to assess speech intelligibility in noise. International Journal of Audiology, 53(10), 760-763.

Huarte, A. (2008). The Castilian Spanish hearing in noise test. International Journal of Audiology, 47(6), 369-370.

Hudgins, C. V., Hawkins, J. E., Kaklin, J. E., \& Stevens, S. S. (1947). The development of recorded auditory tests for measuring hearing loss for speech. The Laryngoscope, 57(1), 57-89.

Jansen, S., Luts, H., Wagener, K. C., Kollmeier, B., Del Rio, M., Dauman, R., et al. (2012). Comparison of three types of French speech-in-noise tests: A multi-center study. International Journal of Audiology, 51(3), 164-173.

Kalikow, D. N., Stevens, K. N., \& Elliott, L. L. (1977). Development of a test of speech intelligibility in noise using sentence materials with controlled word predictability. The Journal of the Acoustical Society of America, 61(5), 1337-1351.

Kar Quar, T., Zms Mukari, S., Alaudin Abdul Wahab, N., Abdul Razak,
R., Omar, M., \& Maamor, N. (2008). The Malay hearing in noise test. International Journal of Audiology, 47(6), 379-380.

Kelly, H., Lin, G., Sankaran, N., Xia, J., Kalluri, S., \& Carlile, S. (2016). Development and evaluation of a mixed gender, multi-talker matrix sentence test in Australian English. International Journal of Audiology, 2016 Oct 19, 1-7 [Epub ahead of print]. http://dx.doi.org/10.1080/14992 027.2016 .1236415 .

Killion, M. C. \& Niquette, P. A. (2000). What can the pure-tone audiogram tell us about a patient's SNR loss? The Hearing Journal, 53(3), 46-48.

Killion, M. C., Niquette, P. A., Gudmundsen, G. I., Revit, L. J., \& Banerjee, S. (2004). Development of a quick speech-in-noise test for measuring signal-to-noise ratio loss in normal-hearing and hearing-impaired listeners. The Journal of the Acoustical Society of America, 116(4), 23952405.

Kollmeier, B., Warzybok, A., Hochmuth, S., Zokoll, M. A., Uslar, V., Brand, T., et al. (2015). The multilingual matrix test: Principles, applications, and comparison across languages: A review. International Journal of Audiology, 54(Suppl 2), 3-16.

Lee, J. H., Jo, S. J., Kim, J. S., Jang, H. S., Lim, D. W., \& Lee, K. W. (2010). Korean speech audiometry. Seoul: Hakjisa.

Lolov, S. R., Raynov, A. M., Boteva, I. B., \& Edrev, G. E. (2008). The Bulgarian hearing in noise test. International Journal of Audiology, 47(6), 371-372.

Moon, S. K., Kim, S. H., Mun, H. A., Jung, H. K., Lee, J. H., Choung, Y. H., et al. (2008). The Korean hearing in noise test. International Journal of Audiology, 47(6), 375-376.

Myhrum, M. \& Moen, I. (2008). The Norwegian hearing in noise test. International Journal of Audiology, 47(6), 377-378.

Nielsen, J. B. \& Dau, T. (2011). The Danish hearing in noise test. International Journal of Audiology, 50(3), 202-208.

Nilsson, M., Soli, S. D., \& Sullivan, J. A. (1994). Development of the Hearing in Noise Test for the measurement of speech reception thresholds in quiet and in noise. The Journal of the Acoustical Society of America, 95(2),1085-1099.

Øygarden, J. (2009). Norwegian speech audiometry (unpublished doctoral dissertation). Norwegian University of Science and Technology, Faculty of Arts, Trondheim.

Ozimek, E., Warzybok, A., \& Kutzner, D. (2010). Polish sentence matrix test for speech intelligibility measurement in noise. International Journal of Audiology, 49(6), 444-454.

Puglisi, G. E., Warzybok, A., Hochmuth, S., Visentin, C., Astolfi, A., Prodi, N., et al. (2015). An Italian matrix sentence test for the evaluation of speech intelligibility in noise. International Journal of Audiology, 54 (Suppl 2), 44-50.

Shiroma, M., Iwaki, T., Kubo, T., \& Soli, S. (2008). The Japanese hearing in noise test. International Journal of Audiology, 47(6), 381-382.

Silverman, S. R. \& Hirsh, I. J. (1955). Problems related to the use of speech in clinical audiometry. The Annals of Otology, Rhinology, and Laryngology, 64(4), 1234-1244.

Soli, S. D. \& Wong, L. L. (2008). Assessment of speech intelligibility in noise with the hearing in noise test. International Journal of Audiology, 47(6), 356-361.

Vaillancourt, V., Laroche, C., Mayer, C., Basque, C., Nali, M., Eriks-Brophy, A., et al. (2005). Adaptation of the HINT (hearing in noise test) for adult Canadian Francophone populations. International Journal of Audiology, 44(6), 358-369.

Vaillancourt, V., Laroche, C., Mayer, C., Basque, C., Nali, M., Eriks-Brophy, A., et al. (2008). The Canadian French hearing in noise test. International Journal of Audiology, 47(6), 383-385.

Wagener, K. (2004). Factors influencing sentence intelligibility in noise. Oldenburg: BIS Verlag.

Wagener, K., Brand, T., \& Kollmeier, B. (1999a). Entwicklung und evaluation eines satztests für die deutsche sprache II: Optimierung des oldenburger satztests. Zeitschrift für Audiologie/Audiological Acoustics, $38,44-56$. 
Wagener, K., Brand, T., \& Kollmeier, B. (1999b) Entwicklung und evaluation eines satztests für die deutsche sprache III: Evaluation des oldenburger satztests. Zeitschrift für Audiologie/Audiological Acoustics, 38, 86-95.

Wagener, K., Kühnel, V., \& Kollmeier, B. (1999c) Entwicklung und evaluation eines satztests für die deutsche sprache I: Design des oldenburger satztests. Zeitschrift für Audiologie/Audiological Acoustics, 38, 4-15.

Wagener, K., Josvassen, J. L., \& Ardenkjaer, R. (2003). Design, optimization and evaluation of a Danish sentence test in noise. International Journal of Audiology, 42(1), 10-7.

Warzybok, A., Zokoll, M., Wardenga, N., Ozimek, E., Boboshko, M., \& Kollmeier, B. (2015). Development of the Russian matrix sentence test. International Journal of Audiology, 54(Suppl 2), 35-43.

Wong, L. L. (2008). The Cantonese hearing in noise test. International Journal of Audiology, 47(6), 388-390.

Wong, L. L. \& Huang, V. (2008). The Taiwanese Mandarin hearing in noise test. International Journal of Audiology, 47(6), 391-392.
Wong, L. L. \& Soli, S. D. (2005). Development of the Cantonese hearing in noise test (CHINT). Ear and Hearing, 26(3), 276-289.

Wong, L. L., Soli, S. D., Liu, S., Han, N., \& Huang, M. W. (2007). Development of the Mandarin hearing in noise test (MHINT). Ear and Hearing, 28(2 Suppl), 70S-74S.

Yi D. (2016). Study on the construction and optimization of the Korean matrix sentence materials (unpublished master's thesis). Hallym University of Graduate Studies, Seoul.

Zokoll, M. A., Fidan, D., Türkyılmaz, D., Hochmuth, S., Ergenç, İ., Sennaroğlu, G., et al. (2015). Development and evaluation of the Turkish matrix sentence test. International Journal of Audiology, 54(Suppl 2), 51-61.

Zokoll, M. A., Warzybok, A., Carroll, R., Hochmuth, S., \& Kreisman, B. (2013). Design, Optimierung und Evaluation eines Amerikanisch Englischen Matrix Test. (Poster) 16th Congress of the German Society of Audiology. Germany, Rostock. 